\title{
Revisión sistemática sobre la aplicación y efectividad de tratamientos basados en mindfulness en personas con discapacidad intelectual
}

\author{
Systematic review on the application and effectiveness of treatments \\ based on mindfulness in people with intellectual disabilities
}

\begin{abstract}
Resumen
El objetivo del presente trabajo ha sido realizar una revisión sistemática de los estudios científicos que han aplicado tratamientos psicológicos basados en mindfulness a población con discapacidad intelectual para profundizar en la evidencia disponible respecto a su aplicabilidad y eficacia. Para ello se realizó una búsqueda bibliográfica en diferentes bases de datos desde julio de 2015 hasta mayo de 2016 y se utilizaron referencias comprendidas entre los años 2000 y 2015. Se seleccionaron y analizaron trece artículos que cumplían con los criterios de inclusión. Los resultados obtenidos indicaron que las terapias basadas en mindfulness parecen influir positivamente en las conductas y emociones de personas con discapacidad intelectual límite, media o moderada, con una edad superior a dieciséis años. Estos hallazgos, sin embargo, deben ser interpretados con cautela debido a las limitaciones metodológicas identificadas en los estudios revisados, siendo necesario seguir investigando y mejorando los diseños de las investigaciones, para poder corroborar los resultados iniciales.
\end{abstract}

\section{Palabras clave}

Discapacidad intelectual, mindfulness, terapia, revisión sistemática.

\section{Abstract}

The aim of this work was to carry out a systematic review of scientific studies that have applied psychological mindfulness-based treatments on people with intellectual disabilities to delve into the available evidence regarding their applicability and efficiency. Therefore, a bibliographic research was carried out for the different data bases from July 2015 to May 2016 and different references were taken between 2000 and 2015. Thirteen relevant articles were chosen and analyzed. The reported findings suggested that the mindfulness-based therapies seems to have a positive influence on the behavior and emotions of people over sixteen with borderline, medium and moderate intellectual disabilities. However, these findings should be carefully interpreted due to the methodological limitations identified in the reviewed studies, and it is necessary to continue investigating and improving research designs in order that they can confirm the initial results.
\end{abstract}

\section{Keywords}

Intellectual disability, mindfulness, therapy, systematic review.

\author{
Rosa Calderón Vicente \\ $<$ rosacv90@gmail.com> \\ Fundación Personas. España
}

\section{Cristina Caballo Escribano} <crisca@usal.es>

Universidad de Salamanca. España

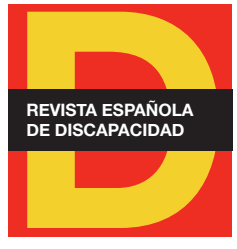

Para citar:

Calderón, R. y Caballo, C. (2018): "Revisión sistemática sobre la aplicación y efectividad de tratamientos basados en mindfulness en personas con discapacidad intelectual". Revista Española de Discapacidad, 6 (I): 49-74.

Doi: <https://doi.org/10.5569/23405104.06.01.03>

Fecha de recepción: 07-08-2017 Fecha de aceptación: 10-01-2018 


\section{Introducción}

En España aproximadamente un 8,5\% de la población tiene alguna discapacidad. Esto es, según la encuesta de Discapacidad, Autonomía personal y situaciones de Dependencia elaborada por el Instituto Nacional de Estadística en 2008, 3,8 millones de personas (Esparza y Abellán, 2008). En concreto, como indican Verdugo et al. (2014), la discapacidad intelectual es una de las de mayor prevalencia, suponiendo aproximadamente entre un $1 \%$ y un $2 \%$ de la población general. Más específicamente, la prevalencia de la discapacidad intelectual grave es de seis por cada mil personas (APA, 2013).

Al hablar de discapacidad intelectual nos referimos a una condición caracterizada por limitaciones significativas tanto en el funcionamiento intelectual como en la conducta adaptativa manifestada en diferentes habilidades adaptativas conceptuales, sociales y prácticas que aparece antes de los 18 años (Schalock et al., 2010). Estas limitaciones complican su interacción con un entorno generalmente poco adaptado a las mismas.

Así, como plantea la Asociación Americana de Discapacidades Intelectuales (AAID, 2010), la discapacidad intelectual se concibe como un estado de funcionamiento que es resultado de la interacción de la persona, con un funcionamiento intelectual y adaptativo limitado, con el entorno. De ello se extrae una premisa principal que implica que, si se mantienen los apoyos personalizados apropiados durante un largo periodo, el funcionamiento en la vida de la persona con discapacidad intelectual generalmente mejorará (Schalock et al., 2010).

\subsection{Discapacidad intelectual y comorbilidad}

Es importante destacar, además, que las personas con discapacidad intelectual presentan entre tres y cuatro veces más trastornos mentales, neurológicos, médicos y físicos que la población general (APA, 2013). Las condiciones asociadas conllevan a menudo graves problemas de comunicación, motores o sensoriales que van a dificultar el proceso de evaluación de la discapacidad intelectual, de la misma manera que las limitaciones derivadas de la discapacidad intelectual pueden suponer una barrera importante a la hora de expresar síntomas característicos de las condiciones asociadas, dificultándose la detección de las mismas (Verdugo et al., 2014). Por otra parte, algunos estudios hablan de la posibilidad de un diagnóstico ensombrecido (Reiss, 2000) debido a que, durante el proceso, los síntomas de los trastornos mentales pueden ser considerados una consecuencia de la discapacidad intelectual y, cuando es así, el diagnóstico se pierde (Whitaker y Read, 2006).

Pese a la dificultad de la realización de los diferentes diagnósticos, los estudios de prevalencia han mostrado de forma consistente que entre un 20 y un $40 \%$ de las personas con discapacidad intelectual también tienen algún tipo de trastorno mental (Bouras et al., 1999; Cooper et al., 2007), prevalencia superior a la encontrada en población con un funcionamiento intelectual normal (Chaplin, 2004). En concreto, Whitaker y Read (2006), presentan evidencias de una mayor prevalencia en niños con discapacidad intelectual que en sus iguales sin discapacidad intelectual y de que tanto en niños como en adultos con discapacidad intelectual grave también es mayor que en personas con discapacidad intelectual media o sin discapacidad intelectual. Los trastornos de personalidad también se dan con mayor frecuencia en personas con discapacidad intelectual (0,2\%-3,9\%) que en personas sin esta condición. Según los mismos autores, 
los datos respecto al trastorno depresivo mayor son contradictorios, situando su prevalencia en personas con discapacidad intelectual en igual o menor rango que en personas sin tal condición. Con respecto a los trastornos de ansiedad, Reid et al. (2011) documentan una incidencia de entre un 3,8 \% y un 15,9\% en personas con discapacidad intelectual, evidenciando una prevalencia mayor que en personas sin discapacidad intelectual. Según el DSM-5 también es frecuente la comorbilidad de la discapacidad intelectual con los trastornos del espectro autista, el trastorno por déficit de atención e hiperactividad, el trastorno bipolar, el de movimientos estereotipados y el trastorno del control de impulsos (APA, 2013).

Las personas con discapacidad intelectual pueden mostrar además conductas agresivas y disruptivas como el daño hacia otros (heteroagresión), hacia sí mismos (autoagresión), la destrucción de propiedades (APA, 2013), así como las rabietas o los gritos (McClintock et al., 2003). Una conducta problemática es aquella que por su intensidad, duración o frecuencia afecta negativamente al desarrollo personal del individuo, así como sus oportunidades de participación en la comunidad (Emerson, 1995). Generalmente, estas conductas tienen una función para la persona que las desempeña y exigen un sobreesfuerzo del sistema de apoyos para atender adecuadamente las necesidades del individuo (Canal y Martín, 2003). Diversos estudios muestran que la prevalencia de los problemas de conducta, tanto en niños como en adultos con discapacidad intelectual, es mayor que en sus iguales sin discapacidad intelectual (Whitaker y Read, 2006). En concreto, cuando se considera la agresión física, la prevalencia oscila entre un $3 \%$ hasta más del $50 \%$, dependiendo de diversas variables (Allen, 2000; Tyrer et al., 2006). La relativa a las conductas autolesivas se encuentra entre un $1,7 \%$ (Rojahn, 1986) y un $41 \%$ (Saloviita, 2000).

Cabe resaltar que los problemas de conducta en personas con discapacidad intelectual son consecuencia de la interacción de múltiples factores, tales como el malestar somático fruto de enfermedades médicas, la incapacidad para comunicarse o los problemas emocionales asociados a la aparición de mayor cantidad de eventos vitales relacionados, en ocasiones, con la exclusión y la discriminación social, el estigma externo e interno, la necesidad de apoyos sociales, físicos y emocionales, así como el hecho de tener menor habilidad cognitiva, la edad de cada persona o el nivel de discapacidad (Idusohan-Moizer et al., 2015).

A menudo las personas que presentan comportamientos problemáticos tienen un desarrollo deficiente de habilidades sociales, habilidades de autocontrol y habilidades para participar en actividades ocupacionales (Dunlap y Kern, 1993). A su vez, las personas que carecen de repertorios comportamentales adecuados tienen más dificultades para lograr sus deseos o satisfacer sus necesidades personales, lo que puede llevar a la frustración. De la experiencia de frustración parten muchos de los episodios de comportamiento problemático, los cuales constituyen el medio para lograr los propósitos y el procedimiento que permite reducir la frustración que sienten (Canal y Martín, 2003). Además de la frustración, la ira (estado emocional que implica una repuesta de activación) es otra variable que puede darse con frecuencia en personas con discapacidad intelectual y que, a menudo, está asociada al desarrollo de conductas agresivas (Del Vecchio y O’Leary, 2004; Willner, 2007) y a distintas formas de psicopatología en ámbitos institucionales y comunitarios (Novaco, 2010).

Por último, el amplio abanico de comportamientos a los que nos referimos cuando hablamos de problemas de conducta puede variar en forma e intensidad, pero siempre tiene una serie de consecuencias negativas, tanto inmediatas (como, por ejemplo, daños físicos) como a largo plazo (por ejemplo, el abuso, abandono, un tratamiento inapropiado o la exclusión social), e interfieren en la calidad de vida de la persona que las manifiesta 
y de familiares o profesionales que conviven con él o ella. Además, en muchas ocasiones llevan a la persona a emplazamientos educativos y residenciales muy restringidos, impidiendo o dificultando su integración en contextos comunitarios o afectando a la vida cotidiana dentro de la familia, al acceso al empleo y a la satisfacción y autorrealización personal (Canal y Martín, 2003). Los estudios apuntan a que la prevalencia de los comportamientos agresivos es mayor en las instituciones (35\%) que en la comunidad (17\%) (Taylor, 2002).

\subsection{Tratamientos para abordar la problemática asociada a la discapacidad intelectual}

De lo descrito previamente se deduce la necesidad de proporcionar apoyo a las personas con discapacidad intelectual también en este ámbito, identificando primero las necesidades de apoyo y planificando, a partir de tal identificación, la prestación de los apoyos requeridos a fin de que ese patrón de comportamiento problemático - que no es inherente a la condición de discapacidad-y las emociones asociadas no interfieran en la consecución de resultados positivos en relación con la calidad de vida, el funcionamiento y el bienestar.

Habitualmente, se utilizan intervenciones farmacológicas y psicológicas o la combinación de ambas como abordajes de primera elección para eliminar problemas emocionales y conductuales.

Se estima que entre un $14 \%$ y un $30 \%$ de personas con discapacidad intelectual son medicadas con psicofármacos para manejar sus conductas agresivas en ausencia de otro trastorno psiquiátrico diagnosticado (Clarke et al., 1990). Sin embargo, la base empírica existente para demostrar su efectividad es limitada (Deb et al., 2007). Revisiones sistemáticas realizadas por Brylewski y Duggan (1999) y Matson et al. (2000), indican que existe escasa evidencia sobre la efectividad de los psicofármacos como tratamiento de primera elección para reducir las agresiones en personas con discapacidad intelectual.

Por su parte, los tratamientos psicológicos también son utilizados para el tratamiento de los trastornos mentales y los problemas de conducta comórbidos en las personas con discapacidad intelectual.

Las intervenciones conductuales, aplicadas por psicólogos, enfermeras y cuidadores especializados se han utilizado ampliamente en los centros e instituciones (Bhaumik et al., 2011). Se consideran tratamientos bien establecidos para problemas conductuales como las agresiones a otros, la autolesión o la destrucción de propiedades en personas con discapacidad intelectual (Carr et al., 2009; Chowdhury y Benson, 2011; Kurtz et al., 2011), aunque no muestran los mismos resultados en la comunidad (Bhaumik et al., 2011). En la actualidad, una de las principales intervenciones es el apoyo conductual positivo (ACP), que consiste en un conjunto de técnicas destinadas a cambiar el entorno para hacer que la conducta problemática sea irrelevante e inútil y, por consiguiente, hacer que la conducta alternativa socialmente adecuada sea más eficaz (Canal y Martín, 2003).

Con respecto a la terapia cognitivo-conductual (TCC), modelo de intervención que domina la investigación psicoterapéutica en personas con discapacidad intelectual actualmente, los resultados sugieren que es efectiva para la ira y las conductas agresivas (Vereenooghe y Langdon, 2013).

Una serie de abordajes terapéuticos, desde hace aproximadamente 20 años, están emergiendo con fuerza en el ámbito de la psicología clínica. Son las terapias de tercera generación o tercera ola de terapias cognitivo-conductuales (Hayes, 2004). 
Dentro de las mismas, uno de los pilares básicos es el denominado mindfulness, término que comenzó a destacar en psicología en los años 80 con el trabajo pionero de Jon Kabat-Zinn, creador de la Terapia de Reducción del Estrés Basada en Mindfulness (Mindfulness-based Stress Reduction Program, MBSR), y que él mismo define como el estado de conciencia que emerge a través de prestar atención en el momento presente, intencionadamente y sin juzgar, al despliegue de la experiencia momento a momento (Kabat-Zinn, 2003). Desde entonces, ha aumentado la investigación y literatura científica en torno a este concepto y su aplicación a la población tanto clínica como no clínica.

En el ámbito clínico se han desarrollado tratamientos psicológicos que se basan o incluyen mindfulness entre sus técnicas y cuyo objetivo general es producir un cambio en la relación de la persona con sus pensamientos y sentimientos, en vez de intentar cambiarlos o eliminarlos (Shapiro y Carlson, 2009). En estos abordajes se enfatiza y se promueve la capacidad de observar y aceptar los eventos internos como son, sin intentar cambiarlos. Algunas de las terapias actuales que cumplen los criterios establecidos por la Asociación Americana de Psicología para ser calificadas como tratamientos bien establecidos y basados en mindfulness son: la Reducción del Estrés Basada en Mindfulness (Kabat-Zinn, 2003), la Terapia Cognitiva Basada en Mindfulness (Segal et al., 2002), la Terapia Dialéctico-Conductual (Linehan, 1993) y la Terapia de Aceptación y Compromiso (Hayes et al., 1999). Cabe destacar que existen dos corrientes teóricas difusas en torno al mindfulness. Una de ellas tiene sus raíces en la psicología y la otra en tradiciones fundamentalmente budistas. Por ejemplo, la Terapia de Aceptación y Compromiso surgió del análisis de la conducta y la teoría de los marcos relacionales, que se ocupa del lenguaje y la cognición (Hayes et al., 2001). En este enfoque, las técnicas psicológicas específicas son utilizadas para mejorar la flexibilidad psicológica de los individuos. Por otro lado, el trabajo de Kabat-Zinn (2003) en la Terapia de Reducción de Estrés Basada en Mindfulness se fundamenta en las enseñanzas budistas de las que deriva su teoría psicológica. Comprende una teoría del comportamiento humano basada en la experiencia de la meditación (Singh et al., 2013c).

En los últimos años se ha producido un aumento de la evidencia científica sobre la efectividad de estos tratamientos basados en mindfulness en un amplio rango de población con problemas físicos y psicológicos (Baer, 2003; Chiesa y Serreti, 2010; Fjorback et al., 2011). De hecho, el National Institute for Health and Care Excellence (NICE) de Inglaterra en su guía del año 2009 recomienda el abordaje mediante la Terapia Cognitiva Basada en Mindfulness para personas con trastorno depresivo y la Terapia Dialéctico-Conductual para personas con trastorno límite de personalidad. Diferentes estudios muestran también que los tratamientos basados en mindfulness son efectivos para los trastornos de ansiedad y, además, se asocian a mejoras en una amplia variedad de síntomas promoviendo, en general, un afrontamiento saludable de situaciones que provocan malestar psicológico y estrés.

\subsection{Mindfulness y discapacidad intelectual}

La limitada gama de psicoterapias basadas en la evidencia disponible para las personas con discapacidad intelectual que presentan trastornos emocionales y conductuales comparada con las que existen para la población sin discapacidad intelectual sugiere la necesidad de incrementar las opciones de tratamiento y, por tanto, el aumento de la investigación y aplicación de terapias que además de ser efectivas en cuanto a la reducción de síntomas supongan una mejora en la relación coste-beneficio de las mismas. Cabría realizarse en este punto la siguiente pregunta: ¿por qué a menudo a las personas con discapacidad intelectual se les niega 
el acceso a una amplia gama de tratamientos potencialmente eficaces que se ofrecen a la población sin discapacidad intelectual? (Idusohan-Moizer et al., 2015). Precisamente, debido a que el mindfulness no se basa en el lenguaje desafía lo que convencionalmente implica cualquier psicoterapia - en especial el componente cognitivo de la TCC - y por ello ha generado especial atención en el campo de la discapacidad intelectual. Un método que puede ser aparentemente simple implica una relación terapéutica y pedagógica en la que pueda darse el proceso de enseñanza y los participantes tengan la capacidad de reconocer y aceptar una sensación o emoción que desencadene una acción, además de la voluntad de la práctica para poder mover el foco de la atención y fomentar así una mejora en su calidad de vida (Flynn, 2012). Otra ventaja importante de este tipo de terapias es que, a diferencia de los procedimientos de conducta tradicionales aplicados en personas con discapacidad intelectual en los que los agentes externos (padres, cuidadores, enfermeras, etc.) son los encargados de administrar las contingencias programadas, con el mindfulness aprenden estrategias de autocontrol con las que la persona regula su propio comportamiento para alcanzar los objetivos deseados por ellos mismos y, además, se salvan los problemas de generalización y mantenimiento propios de las terapias conductual y cognitivo-conductual ya que los usuarios adquieren la capacidad de utilizar los procedimientos en múltiples contextos, proporcionándose autorrefuerzo inmediato ante las conductas encubiertas, los pensamientos y los sentimientos. El papel de los agentes externos sigue siendo importante, pero principalmente para seleccionar las estrategias adecuadas correspondientes a su capacidad cognitiva, enseñar a las personas cómo utilizar esas estrategias, proporcionar apoyo y, en general, ser fuente de motivación (Singh et al., 2013c). Por último y no menos importante, como sucede con la terapia cognitivo-conductual, las técnicas utilizadas en mindfulness deben ser adaptadas a las necesidades de cada persona.

Uno de los procedimientos de mindfulness más utilizados en el campo de la discapacidad intelectual -ante las agresiones y la ira, el abuso del tabaco, los comportamientos sexuales inapropiados y las dificultades emocionales - , ha sido la Meditación en las Plantas de los Pies (Meditation on the Soles of the Feet, SoF). Este procedimiento proporciona estrategias de afrontamiento ante los problemas de conducta enseñando, en primer lugar, a reconocer y aceptar los elementos precursores de la ira y la agresión, es decir, las emociones, sensaciones o pensamientos que activan una respuesta en el individuo y, posteriormente, desviar el foco de la atención desde esa emoción, sensación o pensamiento con una carga emocional a una parte neutra del cuerpo: las plantas de los pies. El resultado de la meditación en las plantas de los pies será el desvanecimiento de la ira o de la carga emocional de la situación (Singh et al., 2013a). Esta técnica se ha utilizado en combinación con otros procedimientos o como base de la terapia en sí misma, y fue inicialmente desarrollada para personas con trastorno mental grave, siendo utilizada posteriormente en población con discapacidad intelectual.

En base a lo descrito previamente, el objetivo de esta revisión es analizar de una manera sistemática los estudios científicos que existen actualmente respecto a la aplicabilidad y eficacia de los tratamientos psicológicos basados en mindfulness en personas con discapacidad intelectual con una edad superior a dieciséis años. Más específicamente se pretende:

Describir el tipo de población con discapacidad intelectual en la que se han aplicado dichos tratamientos.

- Identificar la sintomatología o conductas específicas que se han abordado con tales intervenciones en la población con discapacidad intelectual.

- Especificar el tipo de intervenciones concretas utilizadas, así como las adaptaciones realizadas para su aplicación a la población con discapacidad intelectual 
- Describir la eficacia de tales intervenciones y, en su caso, los beneficios que aporta respecto a las intervenciones más clásicas.

- Analizar la metodología utilizada en cada estudio.

\section{Método}

\subsection{Procedimiento de búsqueda}

Se ha seguido el planteamiento metodológico para desarrollar una revisión sistemática propuesto por Perestelo-Pérez (2013), que ha tenido en cuenta el formato y directrices utilizadas para la elaboración y publicación de las revisiones sistemáticas de la Colaboración Cochrane, la Colaboración Campbell y la declaración PRISMA.

Las publicaciones y artículos utilizados en esta revisión han sido buscados y seleccionados en las siguientes bases de datos: Medline, PsycINFO, Scopus y Psychology And Behavioral Sciencies Collection. En todas ellas se utilizaron varios descriptores combinados: "mindfulness", "intellectual disability", "developmental disabilities", "aggression". No se ha utilizado la palabra terapia para evitar la pérdida de los artículos que, sin usar ese término en su título o abstract, utilizan mindfulness. La búsqueda se ha realizado entre los meses de julio de 2015 y mayo de 2016 y ha incluido artículos publicados desde el año 2000 hasta el año 2015.

El procedimiento de selección de los artículos incluidos en el análisis se basó en una serie de criterios de inclusión establecidos previamente: 1) que utilizasen intervención con mindfulness o con terapias basadas en mindfulness, 2) aplicadas a personas con funcionamiento límite, discapacidad intelectual leve, moderada, grave o profunda y 3) que los participantes tuviesen un rango de edad superior a los 16 años.

Se plantearon como criterios de exclusión del análisis: 1) los estudios que aplicaron mindfulness en participantes con alto funcionamiento, 2) aquellos estudios en los que se aplicó dicha terapia solo en familias y cuidadores, para poder focalizar el análisis de su aplicabilidad y eficacia en la población con discapacidad intelectual y 3) se revisaron únicamente aquellos artículos cuyo texto completo estuviera disponible sin suponer ningún coste económico extra.

\subsection{Análisis y codificación de la información}

La codificación de los estudios incluidos en el análisis se ha realizado a través de la lectura del abstract de los mismos y, en aquellos estudios donde no aparecía suficiente información, se profundizó en la lectura del método y los resultados.

Se codificaron, en primer lugar, los datos relativos a los participantes de cada estudio. La cantidad de sujetos que participó en cada uno de ellos, su género, su edad media, el grado de discapacidad, si tenían o no algún trastorno mental comórbido, si tenían problemas de conducta, si tomaban psicofármacos y, finalmen- 
te, si habían sido institucionalizados en algún centro o unidad hospitalaria. A continuación, se codificaron las intervenciones aplicadas en los estudios: el tipo de terapia, sus características y las posibles adaptaciones realizadas para su aplicación en personas con discapacidad intelectual. Posteriormente, se codificó también la información relativa a la metodología: si eran estudios experimentales controlados o no controlados, si fueron estudios cualitativos y el tipo de diseño empleado en cada estudio. Se codificaron asimismo las diferentes variables resultado utilizadas en cada investigación y, finalmente, los resultados obtenidos en cada estudio mediante la comparación de las puntuaciones medias o el análisis de los tamaños del efecto.

\section{Resultados}

Tras la búsqueda en las diferentes bases de datos se obtuvieron un total de 222 publicaciones potencialmente relevantes, todas ellas publicadas en lengua inglesa, y analizadas para su posterior inclusión. Tras eliminar las referencias duplicadas, quedaron 74 artículos a los cuales se aplicaron los criterios de exclusión e inclusión. Se excluyeron finalmente 61 y han permanecido 13 artículos relevantes en este trabajo. La figura 1 aporta los resultados obtenidos tras el proceso de búsqueda, selección e inclusión de artículos en la presente revisión.

\section{Figura 1. Procedimiento de búsqueda e inclusión de artículos}

Publicaciones identificadas tras la búsqueda en las bases de datos para la revisión $(n=222)$

Publicaciones excluidas tras examinar las referencias duplicadas $(n=148)$

Publicaciones potencialmente relevantes para la revisión $(n=74)$

Publicaciones incluidas en la revisión $(n=13)$

Publicaciones excluidas tras aplicar los criterios de exclusión $(n=61)$

- Artículos de pago $(\mathrm{N}=26)$

- Participantes sin discapacidad intelectual (ej. trastorno del espectro autista con alto funcionamiento, familiares o cuidadores, etc.) ( $\mathrm{N}=27)$

- Estudios que no aplican intervención en mindfulness (ej. revisiones, capítulos de libros, etc.) $(\mathrm{N}=8)$

Fuente: elaboración propia. 
A continuación, se exponen los principales resultados extraídos después del análisis y codificación de los estudios seleccionados.

\begin{tabular}{|c|c|c|c|c|c|c|c|}
\hline Autores & $\begin{array}{c}\mathrm{N} \\
\text { (\%fem) }\end{array}$ & $\begin{array}{l}\text { Edad } \\
\text { Media }\end{array}$ & $\begin{array}{l}\text { Características de } \\
\text { los participantes }\end{array}$ & $\begin{array}{l}\text { Intervención y } \\
\text { adaptaciones }\end{array}$ & $\begin{array}{l}\text { Diseño del } \\
\text { estudio }\end{array}$ & $\begin{array}{l}\text { Variables } \\
\text { resultado }\end{array}$ & Resultados \\
\hline $\begin{array}{l}\text { Miodrag et } \\
\text { al. (2012) }\end{array}$ & $24(54)$ & 28 & $\begin{array}{l}\text { Media Cl=70,7 } \\
\text { DI Límite: } 33 \% \\
\text { DI Media: } 37,5 \% \\
\text { DI Moderada: } \\
\text { 12,5\% } \\
\text { Síndrome de } \\
\text { Prader Willi: } 100 \%\end{array}$ & $\begin{array}{l}\text { - } \text { Programa de } \\
\text { Reducción de } \\
\text { Estrés basado en } \\
\text { Mindfulness } \\
\text { (MBSR) } \\
\text { - Formato grupal } \\
\text { - Adaptación de } \\
\text { instrucciones: } \\
\text { - Sin lenguaje } \\
\text { abstracto } \\
\text { - Repeticiones } \\
\text { - Ayudas visuales } \\
\text { - Uso de ejemplos } \\
\text { reales. }\end{array}$ & $\begin{array}{l}\text { ENC } \\
\text { Pre-post }\end{array}$ & \begin{tabular}{|l} 
Nivel de cortisol \\
Nivel de \\
alfa-amilasa \\
(sAA) \\
Nivel de \\
ansiedad
\end{tabular} & 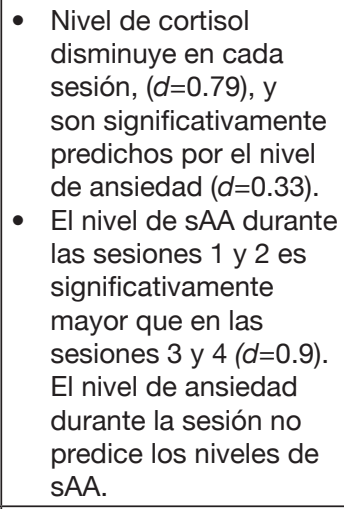 \\
\hline $\begin{array}{l}\text { Singh et } \\
\text { al. (2013b) }\end{array}$ & $3(0)$ & 27 & $\begin{array}{l}\text { DI Media: } 100 \% \\
\text { Sin trastorno } \\
\text { mental asociado. } \\
\text { Nunca } \\
\text { institucionalizado: } \\
100 \% \\
15,17 \text { y } 5 \\
\text { años fumando } \\
\text { respectivamente. }\end{array}$ & $\begin{array}{l}\text { - I. Intención } \\
\text { - II. Observación } \\
\text { consciente de los } \\
\text { pensamientos } \\
\text { - III. Meditación en las } \\
\text { plantas de los pies } \\
\text { (SoF) } \\
\text { - } \text { Formato grupal } \\
\text { - Adaptación a las } \\
\text { necesidades de cada } \\
\text { sujeto. } \\
\text { - Adaptación de } \\
\text { instrucciones: } \\
\text { Lenguaje e idioma. } \\
\end{array}$ & \begin{tabular}{|l|} 
ENC \\
Criterio \\
Cambiante
\end{tabular} & $\begin{array}{l}\text { Cantidad de } \\
\text { cigarrillos } \\
\text { consumidos al } \\
\text { día. }\end{array}$ & $\begin{array}{l}\text { - Sujeto 1: } \\
\text { Disminuyó la cantidad } \\
\text { de } 28 \text { cigarrillos al día a } \\
0 \text { en } 111 \text { días ( } d=1.00) \text {. } \\
\text { - Sujeto } 2 \text { : } \\
\text { Disminuyó la cantidad } \\
\text { de } 33 \text { cigarrillos al día a } \\
0 \text { en } 165 \text { días ( } d=1.00) \text {. } \\
\text { - Sujeto } 3 \text { : } \\
\text { Disminuyó la cantidad } \\
\text { de } 13 \text { a } 0 \text { cigarrillos al } \\
\text { día en } 77 \text { días }(d=1.00) \text {. }\end{array}$ \\
\hline $\begin{array}{l}\text { Singh et } \\
\text { al. (2011a) }\end{array}$ & $3(0)$ & 17 & $\begin{array}{l}\text { DI Media: } 100 \% \\
\text { Síndrome de } \\
\text { Prader Willy: } 100 \% \\
\text { Problemas de } \\
\text { conducta: } 100 \% \\
\text { Sobrepeso: } 100 \% \\
\text { Nunca } \\
\text { institucionalizado: } \\
100 \%\end{array}$ & $\begin{array}{l}\text { - Mindfulness-based } \\
\text { Health Wellness } \\
\text { Program: } \\
\text { - I. Ejercicio físico } \\
\text { - II. Programa } \\
\text { de conciencia } \\
\text { alimentaria. } \\
\text { - III. Atención plena en } \\
\text { la comida. } \\
\text { - IV. Visualización } \\
\text { y etiquetado del } \\
\text { hambre. } \\
\text { - V. Meditación en las } \\
\text { plantas de los pies } \\
\text { (SoF) } \\
\text { - La madre de cada } \\
\text { sujeto se entrena } \\
\text { como terapeuta. }\end{array}$ & \begin{tabular}{|l|} 
ENC \\
Criterio \\
Cambiante
\end{tabular} & \begin{tabular}{|l|} 
Peso en \\
Kilogramos \\
Índice de masa \\
corporal (IMC)
\end{tabular} & $\begin{array}{l}\text { - Sujeto 1: } \\
\text { Pierde 90,77 kg en } 6 \\
\text { años de terapia. } \\
\text { El IMC disminuye de } \\
62.3 \text { a } 24.8 \text { en } 6 \text { años } \\
\text { de terapia. } \\
\text { - Sujeto 2: } \\
\text { Pierde } 19,15 \mathrm{~kg} \text { en } 3 \\
\text { años de terapia. } \\
\text { El IMC disminuye de } \\
34.3 \text { a } 23.3 \text { en } 3 \text { años } \\
\text { de terapia. } \\
\text { Sujeto } 3: \\
\text { Pierde } 24,3 \text { kg en } 3 \\
\text { años de terapia. } \\
\text { El IMC disminuye de } \\
34.6 \text { a } 21.8 \text { en } 3 \text { años } \\
\text { de terapia. }\end{array}$ \\
\hline
\end{tabular}




\begin{tabular}{|c|c|c|c|c|c|c|c|}
\hline Autores & $\begin{array}{c}\mathrm{N} \\
(\% \mathrm{fem})\end{array}$ & $\begin{array}{l}\text { Edad } \\
\text { Media }\end{array}$ & $\begin{array}{l}\text { Características de } \\
\text { los participantes }\end{array}$ & $\begin{array}{l}\text { Intervención y } \\
\text { adaptaciones }\end{array}$ & $\begin{array}{l}\text { Diseño del } \\
\text { estudio }\end{array}$ & $\begin{array}{l}\text { Variables } \\
\text { resultado }\end{array}$ & Resultados \\
\hline $\begin{array}{l}\text { Singh et } \\
\text { al. (2013a) }\end{array}$ & $\begin{array}{l}34 \\
(20)\end{array}$ & 23 & $\begin{array}{l}\text { DI media: } 100 \% \\
\text { Problemas de } \\
\text { conducta: } 100 \% \\
\text { Empleo } \\
\text { remunerado:100\% } \\
\text { Epilepsia: } 9 \% \\
\text { Tratamiento con } \\
\text { psicofármacos: } \\
79 \%\end{array}$ & $\begin{array}{ll}\text { - } & \text { Meditación en las } \\
\text { plantas de los pies } \\
\text { (SoF) } \\
\text { - } \\
\text { Adaptación de } \\
\text { instrucciones. } \\
\text { - } & \text { Modelado } \\
\text { - } & \text { Familiares y } \\
& \text { cuidadores son } \\
\text { entrenados y } \\
\text { enseñan la terapia al } \\
\text { sujeto. } \\
\text { - El terapeuta formado } \\
\text { en mindfulness revisa } \\
\text { las sesiones iniciales. }\end{array}$ & \begin{tabular}{|l|} 
ECA \\
Grupo \\
control \\
lista de \\
espera
\end{tabular} & $\begin{array}{l}\text { Problemas de } \\
\text { conducta: } \\
\text { Agresión física } \\
\text { Agresión verbal }\end{array}$ & $\begin{array}{l}\text { - Las agresiones } \\
\text { físicas disminuyen } \\
\text { significativamente en la } \\
\text { condición experimental } \\
\text { en las fases } 2(d=1.43) \text {, } \\
3 \text { ( } d=1.54) \text { y } 4(d=1.50) \text {. } \\
\text { - Las agresiones verbales } \\
\text { siguen un patrón } \\
\text { similar. El número de } \\
\text { las mismas disminuye } \\
\text { significativamente en } \\
\text { las fases } 2 \text { ( } d=1.35), \\
3 \text { (d=2.16) y } 4(d=1.26) \text {. } \\
\text { No hay diferencias } \\
\text { entre las puntuaciones } \\
\text { de agresión física } \\
\text { y verbal del grupo } \\
\text { control y experimental } \\
\text { durante la primera fase } \\
\text { (entrenamiento de los } \\
\text { padres). }\end{array}$ \\
\hline $\begin{array}{l}\text { Yildiran y } \\
\text { Holt (2014) }\end{array}$ & $6(67)$ & 44 & \begin{tabular}{|l|} 
DI Media- \\
Moderada: \\
$100 \%$ \\
Hospitalizados: \\
$100 \%$ \\
Trastorno mental \\
asociado: $100 \%$ \\
Epilepsia
\end{tabular} & $\begin{array}{l}\text { - I. Atención plena en } \\
\text { la comida. } \\
\text { - II. Body Scan } \\
\text { - III. Atención en la } \\
\text { respiración } \\
\text { - Formato grupal } \\
\text { - Sala adaptada } \\
\text { para estimulación } \\
\text { sensorial: lámparas, } \\
\text { luces y música. }\end{array}$ & $\begin{array}{l}\text { ENC } \\
\text { Cualitativo }\end{array}$ & $\begin{array}{l}\text { Focalizar la } \\
\text { atención } \\
\text { Mejorar } \\
\text { habilidades } \\
\text { Ayudar a otras } \\
\text { personas } \\
\text { Humor } \\
\text { Dificultad de } \\
\text { aprendizaje }\end{array}$ & $\begin{array}{l}\text { - El análisis descriptivo } \\
\text { aporta información } \\
\text { positiva de las cuatro } \\
\text { primeras variables y } \\
\text { recoge, en general, } \\
\text { la opinión de que } \\
\text { el aprendizaje del } \\
\text { procedimiento resultó } \\
\text { complejo a los } \\
\text { participantes. }\end{array}$ \\
\hline $\begin{array}{l}\text { Singh et } \\
\text { al. (2011b) }\end{array}$ & $3(0)$ & 30 & $\begin{array}{l}\text { DI media: } 100 \% \\
\text { Sin trastorno } \\
\text { mental asociado. } \\
\text { Problemas de } \\
\text { conducta: } 100 \% \\
\text { Institucionalizado } \\
\text { alguna vez: } 33,4 \% \\
\text { Empleo } \\
\text { remunerado: } 100 \%\end{array}$ & $\begin{array}{l}\text { - Meditación en las } \\
\text { plantas de los pies } \\
\text { (SoF) } \\
\text { - Formato individual } \\
\text { - Adaptación de } \\
\text { instrucciones: } \\
\text { - Repetición } \\
\text { - El terapeuta es } \\
\text { un compañero } \\
\text { con discapacidad } \\
\text { intelectual } \\
\text { media entrenado } \\
\text { previamente. }\end{array}$ & \begin{tabular}{|l|} 
ENC \\
Línea base \\
múltiple
\end{tabular} & $\begin{array}{l}\text { Problemas de } \\
\text { conducta: } \\
\text { Enfado } \\
\text { Agresión física }\end{array}$ & $\begin{array}{l}\text { - Sujeto 1: } \\
\text { De una media de } 10.63 \\
\text { enfados por semana a } \\
\text { una media de } 3.06 \text { por } \\
\text { semana. } \\
\text { De una media de } 1.13 \\
\text { agresiones por semana } \\
\text { a } 0.38 \text {. } \\
\text { - Sujeto } 2 \text { : } \\
\text { De una media de } 3.86 \\
\text { enfados por semana a } \\
\text { una media de } 1.23 \text { por } \\
\text { semana. } \\
\text { De una media de } 0.86 \\
\text { agresiones por semana } \\
\text { a } 0.11 . \\
\text { Sujeto } 3 \text { : } \\
\text { De una media de } 1.00 \\
\text { enfado por semana a } \\
\text { una media de } 0.30 \text { por } \\
\text { semana. } \\
\text { De una media de } 0.3 \\
\text { agresiones por semana } \\
\text { a 0.08. }\end{array}$ \\
\hline
\end{tabular}




\begin{tabular}{|c|c|c|c|c|c|c|c|}
\hline Autores & $\begin{array}{c}N \\
\text { (\%fem) }\end{array}$ & $\begin{array}{l}\text { Edad } \\
\text { Media }\end{array}$ & $\begin{array}{l}\text { Características de } \\
\text { los participantes }\end{array}$ & $\begin{array}{l}\text { Intervención y } \\
\text { adaptaciones }\end{array}$ & $\begin{array}{l}\text { Diseño del } \\
\text { estudio }\end{array}$ & $\begin{array}{l}\text { Variables } \\
\text { resultado }\end{array}$ & Resultados \\
\hline $\begin{array}{l}\text { Idusohan- } \\
\text { Moizer et } \\
\text { al. (2015) }\end{array}$ & $15(54)$ & 31 & $\begin{array}{l}\text { DI Límite: } 26 \% \\
\text { DI Media: } 54 \% \\
\text { DI Moderada:20\% } \\
\text { Con empleo } \\
\text { remunerado: } 40 \% \\
\text { Trastorno mental } \\
\text { asociado:100\% } \\
\text { Tr. de Ansiedad: } \\
60 \% \\
\text { Tr. Depresivo: } 0 \% \\
\text { Tr. Ansioso- } \\
\text { depresivo:27\% } \\
\text { Tr. Bipolar: } 13 \%\end{array}$ & $\begin{array}{l}\text { - Terapia Cognitiva } \\
\text { Basada en } \\
\text { Mindfulness (MBCT) } \\
\text { - Metáforas y } \\
\text { analogías de } \\
\text { la Terapia de } \\
\text { Aceptación y } \\
\text { Compromiso (ACT) } \\
\text { - Meditación en las } \\
\text { plantas de los pies } \\
\text { (SoF) } \\
\text { - Formato grupal } \\
\text { - Reducción de } \\
\text { ejercicios. } \\
\text { - Ayudas visuales } \\
\text { mediante diapositivas } \\
\text { de PowerPoint con } \\
\text { los puntos clave de } \\
\text { cada sesión. } \\
\text { - Instrucciones escritas } \\
\text { con las tareas para } \\
\text { casa. } \\
\text { - Resúmenes de cada } \\
\text { sesión. } \\
\text { CD con meditaciones } \\
\text { para practicar en } \\
\text { casa. }\end{array}$ & $\begin{array}{l}\text { ECA } \\
\text { Pre-post }\end{array}$ & $\begin{array}{l}\text { Ansiedad } \\
\text { Depresión } \\
\text { Compasión }\end{array}$ & $\begin{array}{l}\text { - Las puntuaciones } \\
\text { en ansiedad fueron } \\
\text { significativamente } \\
\text { menores en el grupo } \\
\text { post-tratamiento } \\
\text { comparados con el } \\
\text { grupo pre-tratamiento } \\
\text { ( } \mathrm{P}<0.01)(\mathrm{r}=-0.70) \text {. } \\
\text { Las puntuaciones post- } \\
\text { tratamiento no difieren } \\
\text { significativamente con } \\
\text { las del seguimiento de } \\
6 \text { semanas }(\mathrm{P}<0.834)(\mathrm{r} \\
=-0.07) \text {. } \\
\text { Las puntuaciones } \\
\text { en depresión fueron } \\
\text { significativamente } \\
\text { menores en el grupo } \\
\text { post-tratamiento } \\
\text { comparados con el } \\
\text { grupo pre-tratamiento } \\
\text { ( } \mathrm{P}<0.05)(r=-0.68) \text {. } \\
\text { Las puntuaciones post- } \\
\text { tratamiento no difieren } \\
\text { significativamente con } \\
\text { las del seguimiento de } \\
6 \text { semanas }(\mathrm{P}<0.13)(\mathrm{r} \\
=-0.48) \text {. } \\
\text { - Las puntuaciones en } \\
\text { compasión fueron } \\
\text { significativamente } \\
\text { mayores en el grupo } \\
\text { post-tratamiento } \\
\text { comparados con el } \\
\text { grupo pre-tratamiento } \\
(\mathrm{P}<0.02)(r=-0.64) \text {. } \\
\text { Las puntuaciones post- } \\
\text { tratamiento no difieren } \\
\text { significativamente con } \\
\text { las del seguimiento de } \\
6 \text { semanas }(\mathrm{P}<0.73)(\mathrm{r} \\
=-0.13) \text {. } \\
\end{array}$ \\
\hline $\begin{array}{l}\text { Singh et } \\
\text { al. (2011c) }\end{array}$ & $1(0)$ & 31 & $\begin{array}{l}\text { DI Media } \\
\text { Institucionalizado } \\
\text { Problemas de } \\
\text { conducta } \\
\text { Sin trastorno } \\
\text { mental asociado } \\
18 \text { años fumando }\end{array}$ & 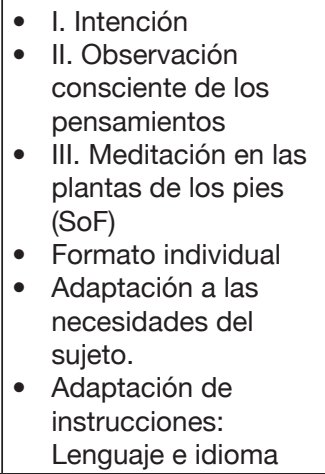 & \begin{tabular}{|l} 
ENC \\
Estudio de \\
caso único \\
Criterio \\
Cambiante
\end{tabular} & $\begin{array}{l}\text { Cantidad de } \\
\text { cigarrillos } \\
\text { consumidos al } \\
\text { día. }\end{array}$ & $\begin{array}{l}\text { - Disminuyó la cantidad } \\
\text { de } 12 \text { cigarrillos al día a } \\
0 \text { en } 82 \text { días. }(d=1.00)\end{array}$ \\
\hline
\end{tabular}




\begin{tabular}{|c|c|c|c|c|c|c|c|}
\hline Autores & $\begin{array}{c}\mathrm{N} \\
\text { (\%fem) }\end{array}$ & $\begin{array}{l}\text { Edad } \\
\text { Media }\end{array}$ & $\begin{array}{l}\text { Características de } \\
\text { los participantes }\end{array}$ & $\begin{array}{l}\text { Intervención y } \\
\text { adaptaciones }\end{array}$ & $\begin{array}{l}\text { Diseño del } \\
\text { estudio }\end{array}$ & $\begin{array}{l}\text { Variables } \\
\text { resultado }\end{array}$ & Resultados \\
\hline $\begin{array}{l}\text { Singh et } \\
\text { al. (2008) }\end{array}$ & $6(0)$ & 29 & $\begin{array}{l}\text { DI Media: } 100 \% \\
\text { Problemas de } \\
\text { conducta: } 100 \% \\
\text { Institucionalizado: } \\
100 \% \\
\text { Tratamiento con } \\
\text { psicofármacos: } \\
33 \% \\
\text { Tr. mental } \\
\text { asociado: } 100 \% \\
\text { Tr. Bipolar: } 17 \% \\
\text { Tr. del Control } \\
\text { de Impulsos y } \\
\text { TDAH: } 17 \% \\
\text { Pedofilia:17\% } \\
\text { Abuso infantil } \\
\text { físico, sexual:32\% } \\
\text { Parafilia: } 17 \%\end{array}$ & $\begin{array}{l}\text { - Meditación en las } \\
\text { plantas de los pies } \\
\text { (SoF) } \\
\text { - Formato individual } \\
\text { - No se llevó a cabo } \\
\text { ninguna adaptación } \\
\text { adicional. }\end{array}$ & $\begin{array}{l}\text { ENC } \\
\text { Línea base } \\
\text { múltiple } \\
\text { Análisis } \\
\text { coste- } \\
\text { beneficio. }\end{array}$ & $\begin{array}{l}\text { Problemas de } \\
\text { conducta: } \\
\text { Agresión física } \\
\text { Agresión verbal } \\
\text { Coste (Dólares) }\end{array}$ & $\begin{array}{l}\text { Reducción de una } \\
\text { media de agresiones } \\
\text { físicas al mes de } 2.33 \text {, } \\
2.33,2.60,2.00,1.29 \text { y } \\
1.00 \text { respectivamente } \\
\text { a } 0 \text { agresiones físicas } \\
\text { al mes por cada } \\
\text { sujeto tras } 27 \text { meses } \\
\text { de intervención con } \\
\text { mindfulness. } \\
\text { - Reducción de una } \\
\text { media de agresiones } \\
\text { verbales al mes de } \\
24.33,13.33,26.00, \\
24.80,20.00 \text { y } 12.14 \\
\text { respectivamente a } \\
3.15,1.63,1.37,2.44, \\
1.93,1.74 \text { agresiones } \\
\text { verbales al mes por } \\
\text { cada sujeto tras } 27 \\
\text { meses de intervención } \\
\text { con mindfulness. } \\
\text { Reducción de los } \\
\text { costes en términos } \\
\text { de los días de baja y } \\
\text { el tratamiento de los } \\
\text { trabajadores heridos de } \\
51,508 \$ \text { a } 2,244 \$ \text {. } \\
\text { Lo que supone una } \\
\text { reducción de } 49,264 \$ \text {. }\end{array}$ \\
\hline $\begin{array}{l}\text { Chapman } \\
\text { y Mitchell } \\
\text { (2013) }\end{array}$ & $60(32)$ & 42 & $\begin{array}{l}\text { DI Media- } \\
\text { Moderada: } 100 \% \\
\text { Con empleo } \\
\text { remunerado:20\% } \\
\text { Problemas de } \\
\text { conducta }\end{array}$ & $\begin{array}{l}\text { - I. Psicoeducación } \\
\text { sobre mindfulness. } \\
\text { - Il. Body-Scan. } \\
\text { - III. Discusión sobre la } \\
\text { experiencia. } \\
\text { - Taller con formato } \\
\text { grupal } \\
\text { - Adaptación de } \\
\text { instrucciones: } \\
\text { Sin lenguaje } \\
\text { abstracto } \\
\text { Lenguaje directo } \\
\text { Folleto con } \\
\text { información clara } \\
\text { sobre mindfulness y } \\
\text { CD con meditaciones } \\
\text { para practicar en } \\
\text { casa. }\end{array}$ & $\begin{array}{l}\text { ENC } \\
\text { Cualitativo }\end{array}$ & $\begin{array}{l}\text { Información y } \\
\text { opinión sobre } \\
\text { los talleres. }\end{array}$ & $\begin{array}{l}\text { El estudio muestra } \\
\text { que es factible } \\
\text { proporcionar talleres } \\
\text { introductorios de } \\
\text { mindfulness a personas } \\
\text { con discapacidad } \\
\text { intelectual y que, en } \\
\text { general, la opinión } \\
\text { sobre los mismos es } \\
\text { positiva. }\end{array}$ \\
\hline
\end{tabular}




\begin{tabular}{|c|c|c|c|c|c|c|c|}
\hline Autores & $\begin{array}{c}\mathrm{N} \\
\text { (\%fem) }\end{array}$ & $\begin{array}{l}\text { Edad } \\
\text { Media }\end{array}$ & $\begin{array}{l}\text { Características de } \\
\text { los participantes }\end{array}$ & $\begin{array}{l}\text { Intervención y } \\
\text { adaptaciones }\end{array}$ & $\begin{array}{l}\text { Diseño del } \\
\text { estudio }\end{array}$ & $\begin{array}{l}\text { Variables } \\
\text { resultado }\end{array}$ & Resultados \\
\hline $\begin{array}{l}\text { Singh et } \\
\text { al. (2007) }\end{array}$ & 3 (33) & 37 & $\begin{array}{l}\text { DI Moderada: } \\
100 \% \\
\text { Tratamiento con } \\
\text { psicofármacos: } \\
100 \% \\
\text { Institucionalizados } \\
\text { alguna vez: } 100 \% \\
\text { Problemas de } \\
\text { conducta: } 100 \% \\
\text { Tr. mental } \\
\text { asociado: } 100 \% \\
\text { Tr. Bipolar: } 33,3 \% \\
\text { Esquizofrenia: } \\
33,3 \% \\
\text { Tr. Psicótico y TEP: } \\
33,4 \%\end{array}$ & 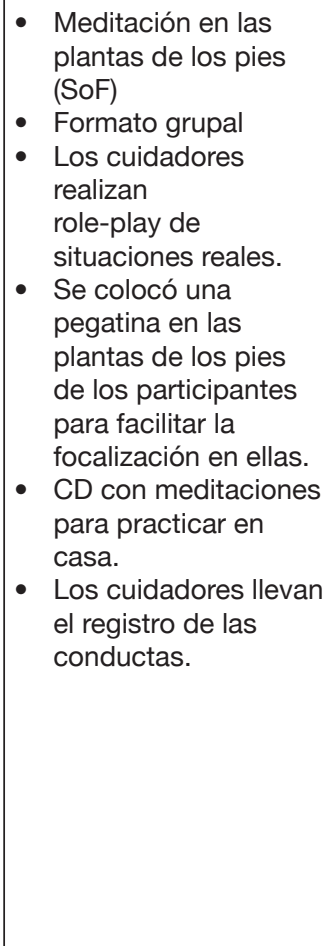 & $\begin{array}{l}\text { ENC } \\
\text { Línea base } \\
\text { múltiple }\end{array}$ & $\begin{array}{l}\text { Problemas de } \\
\text { conducta: } \\
\text { Agresión física. } \\
\text { Agresión verbal. }\end{array}$ & $\begin{array}{l}\text { Los datos muestran } \\
\text { una reducción de los } \\
\text { comportamientos } \\
\text { agresivos: } \\
\text { - Sujeto } 1 \\
\text { De una media de } 5.00 \\
\text { comportamientos } \\
\text { agresivos a la semana } \\
\text { a } 0.8 \text { durante la } \\
\text { intervención y a } 0.1 \\
\text { durante los dos años } \\
\text { de seguimiento. } \\
\text { - Sujeto } 2 \\
\text { De una media de } 3.4 \\
\text { comportamientos } \\
\text { agresivos a la semana } \\
\text { a } 0.6 \text { durante la } \\
\text { intervención y a } 0.3 \\
\text { durante los dos años } \\
\text { de seguimiento. } \\
\text { - Sujeto } 3 \\
\text { De una media de } 2.8 \\
\text { comportamientos } \\
\text { agresivos a la semana } \\
\text { a } 0.3 \text { durante la } \\
\text { intervención y a 0.0 } \\
\text { durante los dos años } \\
\text { de seguimiento. }\end{array}$ \\
\hline $\begin{array}{l}\text { Adkins et } \\
\text { al. (2010) }\end{array}$ & $3(33)$ & 30 & $\begin{array}{l}\text { DI Media: } 100 \% \\
\text { Problemas de } \\
\text { conducta: } 100 \% \\
\text { Tr. mental } \\
\text { asociado: } 100 \% \\
\text { TOC: } 66,7 \% \\
\text { Tr. } \\
\text { Depresivo:33,3\% } \\
\text { Institucionalizados } \\
\text { alguna vez: } 100 \% \\
\text { Con empleo } \\
\text { remunerado:33,3\% } \\
\text { Tratamiento con } \\
\text { psicofármacos: } \\
\text { 33,3\% }\end{array}$ & $\begin{array}{l}\text { - Meditación en las } \\
\text { plantas de los pies } \\
\text { (SoF) } \\
\text { - Formato individual. } \\
\text { - Se entregan } \\
\text { instrucciones } \\
\text { escritas. } \\
\text { - Los cuidadores } \\
\text { actúan como apoyo } \\
\text { para practicar la } \\
\text { técnica fuera de las } \\
\text { sesiones. } \\
\text { - El terapeuta verifica, } \\
\text { comenta y da } \\
\text { feedback a los } \\
\text { participantes cada } \\
\text { semana. }\end{array}$ & $\begin{array}{l}\text { ENC } \\
\text { Línea base } \\
\text { múltiple }\end{array}$ & $\begin{array}{l}\text { Problemas de } \\
\text { conducta } \\
\text { Sujeto 1: } \\
\text { Agresión verbal. } \\
\text { Sujeto 2: } \\
\text { Conducta } \\
\text { disruptiva en el } \\
\text { trabajo. } \\
\text { Sujeto 3: } \\
\text { Agresión física }\end{array}$ & $\begin{array}{l}\text { - Sujeto 1: } \\
\text { Reduce, de una media } \\
\text { de } 4.00 \text { agresiones } \\
\text { verbales a la semana } \\
\text { a } 3.00 \text { tras el } \\
\text { entrenamiento ( } d=1.00) \\
\text { y a } 0.35 \text { durante la } \\
\text { práctica en mindfulness } \\
\text { durante } 28 \text { semanas } \\
\text { ( } d=4.2) \text {. } \\
\text { - Sujeto } 2 \text { : } \\
\text { Reduce, de una media } \\
\text { de } 13.5 \text { conductas } \\
\text { disruptivas en el trabajo } \\
\text { a la semana a } 10.50 \\
\text { en el entrenamiento } \\
\text { ( } d=1.7) \text { y a } 5.58, \text { tras la } \\
\text { práctica en mindfulness } \\
\text { durante } 16 \text { semanas }(d \\
\text { = } 2.02) \text {. } \\
\text { Sujeto } 3 \text { : } \\
\text { Reduce, de una } \\
\text { media de } 12.74 \\
\text { agresiones físicas a la } \\
\text { semana a } 9.00 \text { en el } \\
\text { entrenamiento ( } d=1.02) \text { ( }) \text { y } 1.00 \text { tras la práctica } \\
\text { en mindfulness durante } \\
17 \text { semanas }(d=2.68) \text {. }\end{array}$ \\
\hline
\end{tabular}




\begin{tabular}{|c|c|c|c|c|c|c|c|}
\hline Autores & $\begin{array}{c}\mathrm{N} \\
(\% \mathrm{fem})\end{array}$ & $\begin{array}{l}\text { Edad } \\
\text { Media }\end{array}$ & $\begin{array}{l}\text { Características de } \\
\text { los participantes }\end{array}$ & $\begin{array}{l}\text { Intervención y } \\
\text { adaptaciones }\end{array}$ & $\begin{array}{l}\text { Diseño del } \\
\text { estudio }\end{array}$ & $\begin{array}{l}\text { Variables } \\
\text { resultado }\end{array}$ & Resultados \\
\hline $\begin{array}{l}\text { Singh et } \\
\text { al. (2003) }\end{array}$ & $1(0)$ & 27 & $\begin{array}{l}\text { DI Media } \\
\text { Tr. mental } \\
\text { asociado: } \\
\text { Tr. de Conducta } \\
\text { Tr. Psicótico } \\
\text { Problemas de } \\
\text { conducta } \\
\text { Tratamiento con } \\
\text { psicofármacos } \\
\text { Institucionalizado } \\
\text { alguna vez }\end{array}$ & $\begin{array}{l}\text { - Meditación en las } \\
\text { plantas de los pies } \\
\text { (SoF) } \\
\text { - Formato individual } \\
\text { - Role-play } \\
\text { - Práctica en casa }\end{array}$ & \begin{tabular}{|l|} 
ENC \\
Estudio de \\
caso único \\
Pre-Post.
\end{tabular} & $\begin{array}{l}\text { Problemas de } \\
\text { conducta: } \\
\text { Agresión física } \\
\text { Agresión verbal } \\
\text { Uso } \\
\text { inmediato de } \\
\text { psicofármacos } \\
\text { Contención } \\
\text { física } \\
\text { Daños a } \\
\text { cuidadores o } \\
\text { compañeros } \\
\text { Actividades } \\
\text { sociales }\end{array}$ & $\begin{array}{l}\text { - Agresión física: de } \\
\text { una media de } 15.4 \\
\text { a } 2.0 \text { durante la } \\
\text { intervención y de } 0 \text { en } \\
\text { el seguimiento. } \\
\text { - Agresión verbal: de } \\
\text { una media de } 10.0 \\
\text { a } 2.1 \text { durante la } \\
\text { intervención y de } 0.0 \text { en } \\
\text { el seguimiento. } \\
\text { - Uso inmediato de } \\
\text { psicofármacos: de } \\
\text { una media de } 12.2 \\
\text { a } 0.8 \text { durante la } \\
\text { intervención y de } 0.0 \text { en } \\
\text { el seguimiento. } \\
\text { Contención física: de } \\
\text { una media de } 10.4 \text { a } 0.0 \\
\text { durante la intervención } \\
\text { y el seguimiento. } \\
\text { Daños a cuidadores: de } \\
\text { una media de } 9.2 \text { a } 0 \\
\text { durante la intervención } \\
\text { y el seguimiento. } \\
\text { Daños a compañeros: } \\
\text { de una media de } \\
8.6 \text { a } 0.6 \text { durante la } \\
\text { intervención y a } 0.0 \text { en } \\
\text { el seguimiento. } \\
\text { Actividad social: de } \\
0 \text { a } 43.0 \text { durante la } \\
\text { intervención y }>100 \\
\text { durante el seguimiento. }\end{array}$ \\
\hline
\end{tabular}

Fuente: elaboración propia.

\subsection{Participantes}

Los 13 estudios que cumplieron los criterios de inclusión tenían una muestra diversa de participantes. Los programas basados en mindfulness utilizados en los estudios se han aplicado en un total de participantes de $\mathrm{N}=162$ de los cuales 62 eran mujeres. Nueve de los estudios aplicaron la intervención a un pequeño número de participantes de entre uno y seis sujetos. Los otros cuatro estudios tenían muestras más numerosas: 15 fueron los sujetos que participaron en el experimento de Idusohan-Moizer et al. (2015); 24 sujetos participaron en el estudio de Miodrag et al. (2012) y 34 sujetos en el de Singh et al. (2013a). El experimento que tiene una muestra mayor de sujetos n=60 (Chapman y Mitchell, 2013) no realizó una descripción exhaustiva de la misma y para el análisis de los resultados seleccionó tan solo a seis de los participantes de la muestra.

Los sujetos tenían edades comprendidas entre los 16 y los 44 años (media=29,5). Todos los participantes fueron diagnosticados con algún tipo de discapacidad intelectual. Concretamente, el nivel especificado de discapacidad intelectual más estudiado ha sido el de discapacidad intelectual media en un 84 \% de los 
participantes ( $n=137)$, seguido de discapacidad intelectual moderada en un $8 \%$ de los participantes $(n=13)$ $y$, por último, se han clasificado un $7 \%$ de los sujetos dentro del funcionamiento intelectual límite $(n=12)$. Además de esta condición, el $17 \%$ de los participantes $(n=27)$ tenían diagnosticado Síndrome de PraderWilli. La terapia fue aplicada en un $23 \%$ de participantes que no tenían ningún trastorno mental añadido $(\mathrm{n}=37)$ y en un $21 \%$ de pacientes que sí tenían un diagnóstico mental comórbido ( $\mathrm{n}=34)$. En el $56 \%$ de los participantes no se especificaba tal condición $(n=91)$. El trastorno comórbido que más ha aparecido es el trastorno de ansiedad ( $n=10)$, seguido del trastorno ansioso-depresivo $(n=4)$ y del trastorno bipolar $(n=4)$. Otros trastornos mentales que aparecieron en los estudios son: trastorno depresivo $(n=3)$, trastorno obsesivo-compulsivo $(n=2)$, esquizofrenia $(n=2)$, trastorno psicótico $(n=2)$, trastorno de personalidad $(n=1)$, trastorno del espectro autista $(n=1)$ y trastornos sexuales como parafilia, pedofilia y abuso físico y sexual infantil $(n=5)$. Del total de los participantes: el $51 \%$ tenía problemas de conducta $(n=114)$, el $13 \%$ había sido institucionalizado alguna vez $(n=21)$, el $20 \%$ se encontraba en tratamiento con psicofármacos durante la investigación ( $n=33$ ) y el $35 \%$ tenía, en el momento del estudio, empleo remunerado ( $n=56)$.

\subsection{Tipo de intervención, formato y adaptaciones}

En cuanto a la intervención que se llevó a cabo, el procedimiento basado en mindfulness implementado en un $62 \%$ de los estudios (Adkins et al., 2010; Singh et al., 2013a; Singh et al., 2007; Singh et al., 2008; Singh et al., 2011b; Singh et al., 2003) fue la Meditación en las Plantas de los Pies. Dos de ellos (Singh et al., 2013b; Singh et al., 2011c) utilizaron dicho procedimiento junto con el ejercicio de Intención y el ejercicio de la Observación Consciente de los Pensamientos. Un $8 \%$ de los estudios (Miodrag et al., 2012) utilizó el programa de Reducción de Estrés basado en Mindfulness (MBSR). Finalmente, el $30 \%$ de los estudios analizados empleó un procedimiento de ejercicios combinados de mindfulness. En concreto, en el estudio de Idusohan-Moizer et al. (2015) se emplearon ejercicios de la Terapia Cognitiva Basada en Mindfulness (MBCT), metáforas y analogías de la Terapia de Aceptación y Compromiso (ACT) y la Meditación en las Plantas de los Pies, y en el estudio de Singh et al. (2011a) se utilizó la combinación de ejercicio físico, conciencia alimentaria, atención plena en la comida, visualización y etiquetados del hambre y la Meditación en las Plantas de los Pies.

El 39 \% de los estudios (Chapman y Mitchell, 2013; Dendle y Albany, 2015; Miodrag et al., 2012; Singh et al., 2013b; Yildirian y Holt, 2014) implementaron la terapia siguiendo un formato grupal, con un mínimo de tres participantes y un máximo de seis sujetos en cada grupo. El $46 \%$ de los estudios (Adkins et al., 2010; Singh et al., 2007; Singh et al., 2008; Singh et al., 2011b; Singh et al., 2011c; Singh et al., 2003) siguieron un formato individual de la terapia. En el $15 \%$ restante (Singh et al., 2013a; Singh et al., 2011a) se enseñó primero la terapia a los cuidadores y familiares de las personas que participaron en la investigación para que ellos la implementaran de forma individual con cada uno de los participantes.

En la mayoría de los estudios, la persona encargada de enseñar y supervisar la práctica de la terapia fue un terapeuta ayudado en ocasiones por varios asistentes con experiencia en la práctica y la enseñanza de mindfulness. En el estudio de Singh et al. (2011a), la persona encargada de enseñar el programa de Meditación en las Plantas de los Pies fue un compañero de los participantes con discapacidad intelectual media que previamente había sido entrenado en la técnica y que la había puesto en práctica consigo mismo, logrando buenos resultados en sus problemas de comportamiento. 
Todos los estudios salvo uno (Singh et al., 2008) realizaron adaptaciones en los programas para llevarlas a cabo en población con discapacidad intelectual. Esas adaptaciones se realizaron principalmente en las instrucciones, utilizando un idioma y lenguaje adecuados, evitando el uso de conceptos abstractos y empleando un lenguaje directo. Las instrucciones se repitieron las veces necesarias y en algunos estudios (Adkins et al., 2010; Idusohan-Moizer et al., 2013; Sawicka et al., 2015) se entregaron por escrito. También se emplearon ayudas visuales (Miodrag et al., 2010), mediante diapositivas (Idusohan-Moizer et al., 2015) o realizando, con ayuda de los familiares y cuidadores, role-play de situaciones reales para facilitar la evocación de dichos eventos (Singh et al., 2007; Singh et al., 2003). En el estudio de Singh et al. (2007) se utilizó como ayuda externa una pegatina colocada en las plantas de los pies de los participantes para facilitar el movimiento del foco de la atención a esa zona neutral del cuerpo. Se empleó la técnica de modelado de los ejercicios con ayuda de familiares o cuidadores en el estudio de Singh et al. (2012). En tres estudios (Chapman y Mitchell, 2013; Dendle y Albany, 2015; Singh et al., 2007) se entregó un CD con meditaciones para facilitar la práctica en casa. El estudio de Yildirian y Holt (2014) adaptó una sala de la unidad hospitalaria en la que realizó las sesiones para mejorar la estimulación sensorial con lámparas, luces de colores, música e incienso. En todos ellos se adaptó la terapia a las necesidades de cada sujeto y se utilizó a los cuidadores o familiares como fuente de motivación y apoyo externo para la realización de la práctica en mindfulness en el entorno habitual de cada paciente y, en ocasiones, para completar el autorregistro de las variables resultado.

\subsection{Diseños}

En cuanto al diseño de los estudios, once de ellos, es decir un $85 \%$, fueron estudios no controlados. Dos, un $15 \%$, fueron estudios controlados aleatorizados (Idusohan-Moizer et al., 2015; Singh et al., 2013a): el primero de ellos aleatorizó a los participantes a un grupo de tratamiento o a un grupo lista de espera y el otro en dos grupos experimentales de tratamiento. Cuatro estudios (Adkins et al., 2010; Singh et al., 2007; Singh et al., 2008; Singh et al., 2011b) tuvieron un diseño de línea base múltiple a través de los sujetos. Los estudios de Miodrag et al. (2012), Idusohan-Moizer et al. (2015) y Singh et al. (2003) analizaron las puntuaciones de las distintas variables resultado durante las fases de pre-tratamiento y post-tratamiento, siendo el último de ellos un estudio de caso único. Tres estudios (Singh et al., 2013b; Singh et al., 2011a; Singh et al., 2011c) utilizaron un criterio cambiante en la variable resultado: en el primero y el último (que es un estudio de caso único) el criterio cambiante fue la cantidad de cigarrillos consumida y, en el segundo, la cantidad de peso en kilogramos que deseaba perder cada participante. Por último, dos fueron estudios cualitativos en los que se analizó la información obtenida mediante una entrevista semi-estructurada y un cuestionario (Chapman y Mitchell, 2013; Yildirian y Holt, 2014).

\subsection{Variables resultado medidas}

Las variables resultado analizadas en los estudios experimentales se pueden agrupar en cuatro. Por un lado, los estudios de Singh et al. (2013b) y Singh et al. (2011c) analizaron la cantidad de cigarrillos consumidos al día por los participantes. Es decir, analizaron la eficacia del tratamiento para dejar de fumar.

Por otro lado, seis estudios analizaron la eficacia del tratamiento sobre los problemas de conducta de los participantes. Concretamente, cuatro de los estudios (Singh et al. 2007; Singh et al. 2008; Singh 
et al. 2011b; Singh et al. 2013a) analizaron las puntuaciones medias en agresión física y agresión verbal de los participantes. El último de ellos realizó también un análisis sobre el coste-beneficio de la terapia llevada a cabo. El estudio de Adkins et al. (2010), además de estas dos variables, analizó en uno de los sujetos la conducta disruptiva en el trabajo y, el estudio de Singh et al. (2003) analizó además de la agresión física y verbal, el uso de psicofármacos, de contenciones físicas, de daños a los cuidadores y compañeros, y la cantidad de actividades sociales a las que acudía el sujeto experimental. Con la intención de operativizar qué es exactamente lo que han medido estos estudios cuyas variables resultado han sido los problemas de conducta, se incluye a continuación un resumen de lo que dichos estudios han definido como tal. Al hablar de agresión física se han tenido en cuenta los comportamientos como golpear, abofetear, patear, empujar o dar un puñetazo a otra persona, generalmente un familiar o cuidador, que sufre algún tipo de dolor o lesión tras la agresión. Los estudios que incluyen como variable a cuantificar la destrucción intencionada de propiedades (que implica, por ejemplo, cerrar una puerta con violencia o golpear esa puerta o una pared), la definen asimismo como agresión física.

Se describe como agresión verbal cualquier amenaza oral de daño o agresión física y el uso de un lenguaje abusivo, como gritos o maldiciones, hacia otra persona.

En el estudio de Singh et al. (2011b) se utilizó como variable resultado el enfado y se especificó este comportamiento como una excitación emocional autodetectada, que incluía el aumento de la frecuencia cardiaca, rubor facial, cejas fruncidas, apretar los labios y la sensación de calor que aparece cuando la persona se siente menospreciada, provocada negativamente o con incapacidad de hacer frente a las demandas que se le solicitan.

Finalmente, en el estudio de Adkins et al. (2010) se miden las conductas disruptivas en el trabajo. Lo que se cuantifica específicamente son aquellas conductas en las que el sujeto se aleja de su trabajo, se acerca y conversa con sus compañeros mientras trabajan o aquellas verbalizaciones en las que el sujeto dice a sus compañeros que tiene miedo de ser despedido o que ellos trabajan para hacer que su propio trabajo no sea bien visto.

En cuanto a cómo se han cuantificado esos comportamientos, han sido los cuidadores, familiares o los propios individuos quienes los han registrado cada día de diferentes formas, dependiendo de las circunstancias. La fiabilidad media de las evaluaciones o el acuerdo entre los evaluadores (calculado con la fórmula [acuerdos/(acuerdos+desacuerdos)] x 100) fue de más de un $90 \%$ (salvo en el estudio de Adkins et al. (2010) donde no aparece ese dato).

El nivel de ansiedad de los participantes fue otra de las variables resultado analizadas. En el estudio de Miodrag et al. (2012) se analizaron, además de los niveles de ansiedad, los niveles de cortisol y de alfa-amilasa en la saliva de los participantes y en el estudio de Idusohan-Moizer et al. (2015), a mayores de las puntuaciones en ansiedad se evaluaron las puntuaciones en depresión y compasión. Para terminar, la cuarta variable resultado analizada en uno de los estudios experimentales (Singh et al., 2011a) fue el peso de los participantes medido en kilogramos y las puntuaciones en el índice de masa corporal. Los estudios cualitativos analizaron como variable resultado la opinión de los participantes en cuanto a los talleres de mindfulness en los que participaron (Chapman y Mitchell, 2013) y en cuanto a si la terapia les había servido de ayuda para focalizar la atención, mejorar habilidades, ayudar a otras personas, mejorar su humor o si habían encontrado dificultades en su aprendizaje (Yildirian y Holt, 2014). 


\subsection{Eficacia de las intervenciones}

Respecto a la eficacia de los tratamientos, cabe destacar que en todos los estudios experimentales se obtuvieron mejorías en las variables resultado después de la intervención y la práctica de los programas basados en mindfulness, con tamaños del efecto que oscilan entre $d=0.79$ y $d=2.16$ en aquellos estudios donde aparece o puede calcularse dicho estadístico.

Los resultados sobre la cantidad de cigarros consumidos al día mostraron que esta se redujo significativamente a cero tras la intervención, la fase de mantenimiento y durante el seguimiento de tres años en todos los casos analizados. Los resultados arrojaron un tamaño de efecto elevado $(d=1.00)$, por lo que se concluye que la intervención en mindfulness permite dejar de fumar con éxito y sin recaídas.

Los resultados sobre los problemas de conducta también fueron significativos, con tamaños del efecto que oscilan entre $d=1.00$ y $d=4$.2. El estudio de Singh et al. (2013a) muestra que las puntuaciones en las agresiones físicas disminuyeron significativamente en la condición experimental con tamaños del efecto elevados hasta la fase de seguimiento de un año (dfase $2=1.43$, dfase $3=1.54$, dfase4=1.50) y también en las agresiones verbales (dfase2=1.35, dfase3=2.16, dfase4=1.26). El estudio de Adkins et al. (2010) mostró también la reducción significativa en las puntuaciones medias de los problemas de conducta de los participantes tras la fase de entrenamiento y práctica. Concretamente, las agresiones verbales del primer sujeto se redujeron de una media de 4.00 agresiones semanales a una media de 0.35 durante la fase de práctica del procedimiento. La conducta disruptiva del segundo sujeto se redujo de una media de 13.5 conductas disruptivas semanales en el trabajo a una media de 5.58 durante la fase de práctica y, en el tercer sujeto, las agresiones físicas disminuyeron de 12.74 a la semana a 1.00 durante la fase de práctica. En el estudio de Singh et al. (2011b) se redujeron las puntuaciones medias de enfado y de agresión física a niveles próximos a cero durante la fase de práctica de mindfulness de cada sujeto. Además, durante las últimas cuatro y seis semanas de entrenamiento, en dos de los tres participantes no se registraron ni enfado ni agresiones físicas y durante el seguimiento de dos años se registraron eventos ocasionales de enfado, pero ninguna agresión física por parte de los sujetos. En el estudio de Singh et al. (2008), tras veintisiete meses de entrenamiento en el programa de mindfulness, los comportamientos agresivos, tanto las agresiones físicas como las agresiones verbales, disminuyeron a cero en todos los individuos. Además, durante la intervención no fue requerida medicación ni contención física y tampoco se produjeron lesiones en los trabajadores ni en los compañeros. En cuanto al análisis de coste-beneficio, los resultados mostraron una reducción en los costes del 95,7\%, que en dólares supone una cantidad igual a 49.264 \$. En el estudio de Singh et al. (2007), también se redujeron los comportamientos agresivos semanales a valores muy próximos a cero tras la terapia y el seguimiento $(0.1,0.3$ y 0.0 respectivamente). Por último, en el estudio de Singh et al. (2003) la media de agresiones físicas y verbales mensuales se redujeron a valores próximos o igual a cero durante la intervención y el seguimiento y lo mismo sucedía con el uso de psicofármacos y de contenciones físicas para controlar dichas conductas de forma externa. Los daños a los cuidadores fueron eliminados completamente tras la intervención y durante el seguimiento. Los daños a los compañeros se redujeron a 0.6 durante la intervención y a 0.0 durante el seguimiento. Otro dato que arroja este estudio es sobre las actividades sociales realizadas al mes, las cuales aumentaron de 0.00 durante la línea base a una media de 43.00 durante la intervención y a más de 100 durante el seguimiento.

En relación con la variable ansiedad, también los resultados de los estudios indican que la intervención fue eficaz para reducirla, con tamaños del efecto entre $d=0.79$ y $d=0.9$. Así, por ejemplo, los resultados sobre 
los niveles de ansiedad, cortisol y alfa-amilasa en el estudio de Miodrag et al. (2012) registraron reducciones de los mismos: tras la intervención hubo diferencias estadísticamente significativas entre los niveles de cortisol antes y después de cada sesión $(p<.001)$ con un tamaño del efecto elevado $(d=0.79)$ y los niveles de cortisol y ansiedad, autoevaluada mediante el CBCL/6-18, siguieron el mismo patrón a lo largo de las sesiones disminuyendo ambas sus niveles en cada sesión. Por tanto, la ansiedad autoevaluada predecía significativamente los niveles de cortisol $(p=.014)$ aunque con un tamaño del efecto pequeño $(d=0.33)$. En cuanto a los niveles de alfa-amilasa, no parecieron seguir el mismo patrón que los de cortisol: los niveles de alfa-amilasa durante las dos primeras sesiones fueron significativamente mayores que en las sesiones cuatro y cinco, con un tamaño de efecto elevado $(d=0.9)$. Los niveles de ansiedad durante la intervención no predecían los niveles de alfa-amilasa en la saliva, lo que apoyaría la idea de que el cortisol y la alfa-amilasa son indicadores de diferentes componentes del sistema biológico del estrés. En el estudio de IdusohanMoizer et al. (2015) la eficacia del programa se mostró en los resultados obtenidos: por un lado, las puntuaciones en la Escala de Compasión de los grupos post-tratamiento fueron significativamente mayores que en el pre-tratamiento $(p<.02, r=-0.64)$; por otro lado, las puntuaciones en ansiedad obtenidas mediante la Escala Hospitalaria de Ansiedad y Depresión (HADS) en el post-tratamiento fueron significativamente menores que en el pre-tratamiento $(p<0.01, r=-0.70) y$, para terminar, las puntuaciones en depresión obtenidas con la misma escala (HADS) fueron significativamente menores en el post-tratamiento que en el pre-tratamiento $(\mathrm{p}<0.05, \mathrm{r}=-0.67)$.

El estudio de Singh et al. (2011a), el único que queda por analizar de entre los cuantitativos, mostraba reducciones significativas tanto en el peso de los participantes $(99.77 \mathrm{~kg}, 19.15 \mathrm{~kg}$ y $24.3 \mathrm{~kg}$ menos en cada uno de ellos), como en los índices de masa corporal que se modificaron de 62.3, 34.4 y 34.6 a 24.8, 23.3 y 21.8 respectivamente durante la terapia.

El estudio cualitativo de Yildirian y Holt (2014) realizó un análisis temático de las respuestas de los participantes en la entrevista y recogía, en primer lugar, que la experiencia de los participantes encajaba con la finalidad teórica del mindfulness de controlar el foco de la atención y que los participantes reconocieron una serie de habilidades físicas que atribuyeron a la práctica del mindfulness. En segundo lugar, se afirma que la práctica en mindfulness permitió a los participantes pensar en su relación con otras personas y realizar acciones que les ayudasen, y también atribuyeron al procedimiento mejoras en su humor. Por último, algunos de los participantes reconocieron haber dudado de su habilidad para aprender las técnicas. El otro estudio cualitativo recogido en esta revisión (Chapman y Mitchell, 2013), mostró un feedback de los participantes tras los talleres de mindfulness que fue, en general, positivo. Los sujetos valoraron especialmente la posibilidad de poder compartir la experiencia con otras personas y encontraron muy relajante el ejercicio del escaneo corporal. Dos de los participantes lo encontraron útil a la hora de lidiar con fobias, estrés o dificultades sociales como la discriminación. También indicaron que serían de utilidad más sesiones de mindfulness y que dicho programa podría ser beneficioso para otras personas con discapacidad. Para finalizar, tres de los sujetos entrevistados afirmaron haber utilizado el CD que les entregaron en casa. 


\section{Discusión}

Los trece estudios analizados en esta revisión sugieren que las terapias basadas en mindfulness influyen positivamente en las conductas y las emociones de las personas mayores de dieciséis años con discapacidad intelectual límite, media o moderada, mostrando resultados favorables en la reducción de conductas problemáticas, del consumo de tabaco, del peso corporal y de los niveles de ansiedad y depresión. Estas mejoras han sido mantenidas a lo largo de varios años de seguimiento y además suponen beneficios en cuanto a la reducción de los gastos invertidos en paliar los problemas físicos y psicológicos que aparecen en los trabajadores que cuidan a las personas con discapacidad intelectual.

Los estudios sugieren, asimismo, que las terapias basadas en mindfulness benefician a las personas con discapacidad límite, media y moderada mayores de dieciséis años cuando se las enseñan directamente a ellas, pero también cuando se las enseñan a través de sus familiares y cuidadores. El feedback de los participantes demuestra que las terapias basadas en mindfulness son perfectamente aplicables y accesibles a esta población cuando se utilizan instrucciones claras, ejemplos reales y concretos, se alienta y se realiza una práctica continua y cuando, en definitiva, se realizan ciertas adaptaciones en los procedimientos habituales. Los estudios también apuntan a la mejora de los procedimientos cuando los familiares y cuidadores de las personas con discapacidad intelectual participan en los mismos, favoreciendo la motivación y aportando una ayuda externa para el correcto aprendizaje y práctica de los programas y, en ocasiones, como medio para completar los autorregistros de las variables medidas en cada experimento. Se demuestra asimismo que las terapias basadas en mindfulness funcionan cuando son aplicadas tanto en contextos comunitarios como en contextos institucionales.

En definitiva, este análisis nos muestra cierta evidencia de que las terapias basadas en mindfulness pueden ser de utilidad para reducir conductas inapropiadas, poco saludables o emociones negativas manifestadas por personas con discapacidad intelectual.

\subsection{Limitaciones metodológicas}

Al margen de la importancia de estos hallazgos, es necesario considerar las limitaciones metodológicas de las investigaciones y tomar con precaución los resultados. Once de las trece publicaciones analizadas son estudios experimentales no controlados. Los participantes no han sido seleccionados aleatoriamente y no existe una condición de comparación entre grupos experimentales y otros grupos en los que no se apliquen los tratamientos. Por lo tanto, la validez interna de las investigaciones es escasa. Por otro lado, el tamaño de la muestra en nueve de los estudios es muy reducido, lo que dificulta la generalización de los resultados a otras poblaciones e implica una validez externa limitada. La descripción de los sujetos tampoco es exhaustiva en todos los estudios. De hecho, se desconoce la comorbilidad de diagnósticos en un $56 \%$ de los casos. Cabe destacar que los estudios han aplicado las terapias a personas con funcionamiento límite o discapacidad intelectual media y moderada. Ningún estudio ha probado la eficacia en población con discapacidad intelectual grave o profunda y, por tanto, la generalización de los resultados a todos los niveles de discapacidad intelectual se hace imposible. Ocho estudios incluidos en la revisión describieron e implementaron la Meditación en las Plantas de los Pies, el programa basado en mindfulness desarrollado por 
Singh y su equipo que en dos ocasiones se acompañó del ejercicio de la Intención y el de la Observación Consciente de los Pensamientos. El resto describen terapias con una variedad de ejercicios que provienen de los programas de MBSR y MBCT, entre otros. Esto implica que no hay un programa de intervención utilizado sistemáticamente en todas las investigaciones, con el mismo número de sesiones y la misma duración de cada una de ellas que permita replicar los estudios. Además, la enseñanza y evaluación de las terapias basadas en mindfulness debe ser implementada por personas rigurosamente entrenadas y supervisadas regularmente por terapeutas (Baer, 2003), y en la mayoría de los estudios analizados los terapeutas son descritos como personas con experiencia en mindfulness, pero esta característica aparece escasamente definida. Por último, cabe señalar que en la mayoría de los estudios aparecen tan solo las puntuaciones medias de las conductas durante la línea base, el entrenamiento, la práctica y el seguimiento, registradas por uno o varios agentes externos. Tan solo en tres estudios (Idusohan-Moizer et al., 2015; Singh et al., 2013a; Singh et al. 2013b) aparecen estadísticos tan relevantes como el tamaño del efecto - descrito mediante la $d$ de Cohen y el coeficiente de correlación de Pearson $(r)-$ o la potencia.

\subsection{Implicaciones para futuros estudios}

Los estudios revisados en el presente análisis demuestran que las personas con discapacidad intelectual límite, media o moderada pueden aprender y, por tanto, beneficiarse de los procedimientos utilizados en las terapias basadas en mindfulness. Este beneficio a nivel individual, tendría necesariamente una repercusión a nivel social, promoviendo el empoderamiento de las personas con discapacidad intelectual, el derecho a decidir y la igualdad en cuanto al acceso a esta alternativa terapéutica que ya funciona en la población general, así como la posible reducción del gasto público dedicado a la medicación y a la institucionalización de las personas con discapacidad ligado a los problemas de conducta que presentan.

En este punto, planteamos la necesidad de aumentar la investigación en este campo. En concreto, son necesarios más estudios llevados a cabo con un control metodológico más preciso para poder concluir de forma sólida que las terapias basadas en mindfulness son, tal y como se esboza en el análisis, factibles y efectivas en una población en la que se implementan con poca frecuencia terapias novedosas y demostradamente efectivas en población sin discapacidad intelectual. Es necesario realizar estudios controlados y aleatorizados, a ser posible con un grupo control lista de espera que los haga éticamente más correctos y con un tamaño de muestra mayor que en los realizados hasta ahora. Será necesaria una descripción más exhaustiva de los participantes, de las condiciones bajo las que se realiza cada intervención y aplicar ésta en personas con diferentes niveles de discapacidad intelectual. Los próximos estudios han de ser más concisos en cuanto al procedimiento basado en mindfulness utilizado y describir, de forma más rigurosa, el tipo de ejercicios, el número de sesiones, la duración de las mismas y las adaptaciones o posibles ayudas utilizadas en cada caso, y deben también explicitar la cualificación y experiencia del terapeuta que dirija las terapias. Todo ello para obtener datos y estadísticos más robustos que nos permitan atribuir, de una forma más rigurosa, los cambios producidos en el comportamiento, las emociones y la calidad de vida de las personas con discapacidad intelectual a la acción de la terapia basada en mindfulness. 


\section{Conclusión}

Esta revisión sistemática permite alcanzar los objetivos planteados al inicio, primeramente, porque se ha analizado la evidencia empírica existente en los estudios científicos respecto a la aplicabilidad y eficacia de los tratamientos basados en mindfulness en personas con discapacidad intelectual. En segundo lugar, porque se ha profundizado en la descripción de la población a la que se han aplicado los tratamientos, se ha descrito qué tipo de intervenciones y adaptaciones de las mismas se han realizado y sobre qué problemática o sintomatología concreta se han aplicado y se ha analizado la metodología utilizada en los estudios. Por último, y de los beneficios que aporta respecto a las intervenciones más clásicas no ha sido posible, ya que los estudios no realizaron comparaciones rigurosas entre dos o más terapias psicológicas y/o farmacológicas. 
Adkins, A. D. et al. (2010): "Using a mindfulness-based procedure in the community: translating research to practice". Journal of Child and Family Studies, 19 (2): 175-183.

Allen, D. (2000): "Recent research on physical aggression in persons with intellectual disability: an overview". Journal of Intellectual and Developmental Disability, 25 (1): 41-57.

American Psychiatric Association, APA (2013): Diagnostic and Statistical Manual of Mental Disorders: DSM-5 (5 ed.). Washington DC: American Psychiatric Publising.

Baer, R. A. (2003): "Mindfulness as a clinical intervention: A conceptual and empirical review". Clinical Psychology: Science and Practice, 10 (2): 125-143.

Bhaumik, S. et al. (2011): "Psychological treatments in intellectual disability: the challenges of building a good evidence base". The British Journal of Psychiatry, 198 (6): 428-430.

Bouras, N. et al. (1999): "Mental health in mental retardation: The ABC for mental health, primary care and other professionals". Londres: WPA Section of Mental Retardation.

Brylewski, J. y Duggan, L. (1999): "Antipsychotic medication for challenging behaviour in people with learning disability". Journal of Intellectual Disability Research, 43: 360-371.

Canal, R. y Martín, M. V. (2003): Apoyo conductual positivo. Valladolid: Junta de Castilla y León.

Carr, J. E. et al. (2009): "Noncontingent reinforcement is an empirically supported treatment for problem behavior exhibited by individuals with developmental disabilities". Research in Developmental Disabilities, 30 (1): 44-57.

Chapman, M. J. y Mitchell, D. (2013): "Mindfully valuing people now: an evaluation of introduction to mindfulness workshops for people with intellectual disabilities". Mindfulness, 4 (2): 168-178.

Chaplin, R. (2004): "General psychiatry services for adults with intellectual disabilities and mental illness". Journal of Intellectual Disability Research. 48 (1): 1-10.

Chiesa, A. y Serretti, A. (2010): "A systematic review of neurobiological and clinical features of mindfulness meditations". Psychological Medicine, 40 (08): 1239-1252.

Chowdhury, M. y Benson, B. A. (2011): "Use of differential reinforcement to reduce behavior problems in adults with intellectual disabilities: a methodological review". Research in Developmental Disabilities, 32 (2): 383-394.

Clarke, D. J. et al. (1990): "Psychotropic drugs and mental retardation: 1. Disabilities and the prescription of drugs for behaviour and for epilepsy in three residential settings". Journal of Intellectual Disability Research, 34 (5): 385-395.

Cooper, S. A. et al. (2007): "Mental ill-health in adults with intellectual disabilities: prevalence and associated factors". The British Journal of Psychiatry, 190 (1): 27-35.

Deb, S. et al. (2007): "The effectiveness of antipsychotic medication in the management of behaviour problems in adults with intellectual disabilities". Journal of Intellectual Disability Research, 51 (10): 766-777.

Del Vecchio, T. y O'Leary, K. D. (2004): “Effectiveness of anger treatments for specific anger problems: a metaanalytic review". Clinical Psychology Review, 24 (1): 15-34. 
Dunlap, G. y Kern, L. (1993): "Assessment and intervention for children within the instructional curriculum”, en J. Reichle., y D. Wacker. (eds.): Communicative approaches to the management of challenging behavior. Baltimore: Paul H. Brookes.

Emerson, E. (1995): Challenging behaviour: analysis and intervention in people with learning difficulties. Cambrigde: Cambrigde University Press.

Esparza, C. y Abellán, A. (2008): Encuesta de Discapacidad, Autonomía personal y situaciones de Dependencia (EDAD) 2008. Madrid: Instituto Nacional de Estadística.

Fjorback, L. O. et al. (2011): "Mindfulness-Based Stress Reduction and Mindfulness-Based Cognitive Therapy: a systematic review of randomized controlled trials". Acta Psychiatrica Scandinavica, 124 (2): 102-119.

Flynn, A. G. (2012): "Fact or faith?: on the evidence for psychotherapy for adults with intellectual disability and mental health needs". Current Opinion in Psychiatry, 25 (5): 342-347.

Hayes, S. C. (2004): "Acceptance and commitment therapy, relational frame theory, and the third wave of behavioral and cognitive therapies". Behavior therapy, 35 (4): 639-665.

Hayes, S. C. et al. (eds.) (2001): Relational frame theory: A post-Skinnerian account of human language and cognition. Nueva York: Kluwer Academic/Plenum Publishers.

Hayes, S. C. et al. (1999): Acceptance and Commitment Therapy: An experiential approach to behavior change. Nueva York: Guilford Press.

Idusohan-Moizer, H. et al. (2015): "Mindfulness-Based Cognitive Therapy for adults with intellectual disabilities: an evaluation of the effectiveness of mindfulness in reducing symptoms of depression and anxiety". Journal of Intellectual Disability Research, 59 (2): 93-104.

Kabat-Zinn, J. (2003): "Mindfulness-based interventions in context: past, present, and future”. Clinical Psychology: Science and Practice, 10 (2): 144-156.

Kurtz, P. F. et al. (2011): "An analysis of functional communication training as an empirically supported treatment for problem behavior displayed by individuals with intellectual disabilities". Research in Developmental Disabilities, 32 (6): 2935-2942.

Linehan, M. (1993): Cognitive-Behavioral Treatment of Borderline Personality Disorder. Nueva York: Guilford Press.

Matson, J. L. et al. (2000): “Psychopharmacology and mental retardation: a 10 year review (1990-1999)". Research in Developmental Disabilities, 21 (4): 263-296.

McClintock, K. et al. (2003): "Risk markers associated with challenging behaviours in people with intellectual disabilities: a meta-analytic study". Journal of Intellectual Disability Research, 47 (6): 405-416.

Miodrag, N. et al. (2013): "A pilot study of a mindfulness intervention for individuals with Williams Syndrome: physiological outcomes”. Mindfulness, 4 (2): 137-147.

Novaco, R. W. (2010): “Anger and psychopathology”, en Potegal, M. et al. (eds.): International handbook of anger: constitutent and concomitant biological, psychological and social proceses. Nueva York: Springer.

Perestelo-Pérez, L. (2013): "Standard son how to develop and report systematic reviews in Psychology and Health". International Journal of Clinical and Health Psychology, 13: 49-57.

Reid, K. A. et al. (2011): "Prevalence and associations of anxiety disorders in adults with intellectual disabilities". Journal of Intellectual Disability Research, 55 (2): 172-181. 
Reiss, S. (2000): “A mindful approach to mental retardation”. Journal of Social Issues, 56(1), 65-80.

Rojahn, J. (1986): "Self-injurious and stereotypic behavior of noninstitutionalized mentally retarded people: prevalence and classification”. American Journal of Mental Deficiency, 91 (3): 268-276.

Saloviita, T. (2000): "The structure and correlates of self-injurious behavior in an institutional setting". Research in Developmental Disabilities, 21 (6): 501-511.

Schalock, R. L. et al. (2010): Intellectual Disability. Definition, classification and systems of supports. (11.a ed.). Washington, DC: American Association on Intellectual and Developmental Disabilities.

Segal, Z. V. et al. (2002): Mindfulness-based cognitive therapy for depression: a new approach to preventing relapse. Nueva York: Guilford Press.

Shapiro, S. L. y Carlson, L. E. (2009): The Art and Science of Mindfulness: Integrating Mindfulness into Psychology and the Helping Professions. Washington, DC: American Psychological Association.

Singh, N. N. et al. (2013a): "Mindfulness-based treatment of aggression in individuals with mild intellectual disabilities: a waiting list control study". Mindfulness, 4 (2): 158-167.

Singh, N. N. et al. (2013b): "A Mindfulness-based smoking cessation program for individuals with mild intellectual disability”. Mindfulness, 4 (2): 148-157.

Singh, N. N. et al. (2013c). Mindfulness-Based Approaches, en Taylor, J. L. et al. (eds.): Psychological Therapies for Adults With Intellectectual Disabilities. Chichester, Reino Unido: John Wiley \& Sons, Ltd.

Singh, N. N. et al. (2011a): "A Mindfulness-Based Health Wellness Program for Individuals With Prader-Willi Syndrome". Journal of Mental Health Research in Intellectual Disabilities, 4 (2), 90-106.

Singh, N. N. et al. (2011b): "Peer with intellectual disabilities as a Mindfulness-based anger and aggression management therapist". Research in Developmental Disabilities, 32 (6): 2690-2696.

Singh, N. N. et al. (2011c): "Effects of a Mindfulness-based smoking cessation program for an adult with mild intellectual disability”. Research in Developmental Disabilities, 32 (3): 1180-1185.

Singh, N. N. et al. (2008): "Clinical and benefit-cost outcomes of teaching a Mindfulness-based procedure to adult offenders with intellectual disabilities”. Behavior Modification, 32 (5): 622-637.

Singh, N. N. et al. (2007): "Mindfulness training assists individuals with moderate mental retardation to maintain their community placements". Behavior Modification, 31 (6): 800-814.

Singh, N. N. et al. (2003): "Soles of the feet: a Mindfulness-based self-control intervention for aggression by an individual with mild mental retardation and mental illness". Research in Developmental Disabilities, 24 (3): 158-169.

Taylor, J. L. (2002): "A review of the assessment and treatment of anger and aggression in offenders with intellectual disability”. Journal of Intellectual Disability Research, 46 (s1): 57-73.

Tyrer, F. et al. (2006): "Physical aggression towards others in adults with learning disabilities: prevalence and associated factors". Journal of Intellectual Disability Research, 50 (Pt 4): 295-304.

Verdugo, M. A. et al. (2014): “Discapacidad intelectual”, en Expeleta, L. y Toro, J. (eds.): Psicopatología del desarrollo. Madrid: Pirámide.

Vereenooghe, L., y Langdon, P. E. (2013): "Psychological therapies for people with intellectual disabilities: a systematic review and meta-analysis". Research in Developmental Disabilities, 34 (11): 4085-4102. 
Whitaker, S. y Read. S. (2006): "The prevalence of psychiatric disorders among people with intellectual disabilities: an analysis of the literature". Journal of Applied Research in Intellectual Disabilities. 19 (4): 330-345.

Willner, P. (2007): “Cognitive behavioural therapy for people with learning disabilities: focus on anger". Advances in Mental Health and Learning Disabilities, 1 (2): 14-21.

Yildiran, H. y Holt, R. R. (2015): "Thematic analysis of the effectiveness of an inpatient mindfulness group for adults with intelectual disabilities". British Journal of Learning Disabilities, 43 (1): 49-54. 